\title{
Indicadores no contexto da Politica Nacional de Saúde Bucal: uma revisão integrativa
}

I ${ }^{1}$ Pedro Augusto Thiene Leme, ${ }^{2}$ Otília Maria Lúcia Barbosa Seiffert I

Resumo: Trata-se de uma revisão integrativa da literatura sobre os indicadores de serviço relacionados ao campo da odontologia no Brasil. Partiu-se da seguinte questão de pesquisa: os indicadores utilizados em estudos que tiveram como objeto os serviços odontológicos no SUS, são coerentes com o paradigma de promoção de saúde defendido pela Política Nacional de Saúde Bucal ou se restringem ao escopo tradicional de programação dos serviços? A busca foi realizada nas bases de dados Scielo e Bireme, compreendendo artigos revisados por pares publicados entre os anos de 2004 e 2018, resultando em amostra final de 21 artigos. Os artigos foram lidos na íntegra e foram extraídas informaçôes sobre os indicadores utilizados, posteriormente categorizados e analisados. Os resultados evidenciam que, embora a diversidade de indicadores seja capaz de representar aspectos relevantes da realidade, de forma geral desconsideram a categoria do cuidado e atributos do conceito de promoção de saúde, além de existir uma carência de indicadores de resultados, que permitiriam avaliar o impacto da política sobre as demandas dos usuários.

> Palavras-chave: Saúde Bucal. Odontologia. Sistema Único de Saúde. Indicadores de Serviço. Revisão.

\author{
${ }^{1}$ Universidade Estadual de \\ Campinas. Campinas-SP, Brasil \\ (pedroleme3@gmail.com). \\ ORCID: 0000-0003-3894-3189 \\ ${ }^{2}$ Universidade Federal de São \\ Paulo, São Paulo-SP, Brasil \\ (otiliaseiffert@gmail.com). \\ ORCID: 0000-0002-8994-1469
}




\section{Introdução}

A Saúde Bucal, ainda que arbitrariamente adjetivada pelo segundo termo, é componente indissociável da saúde geral, e assim, relaciona-se com a questáo da integralidade nos sistemas de saúde (CHAVES, 2010; PAIM, 1997). O Brasil possui a maior rede de serviços públicos de saúde bucal, de acesso universal no mundo, dentro do Sistema Único de Saúde - SUS (SCHERER, 2015; BRASIL, 2004). A Política Nacional de Saúde Bucal (PNSB) de 2004 inseriu o cirurgião dentista na Estratégia de Saúde da Família (ESF) e nos Centros de Especialidades Odontológicas (CEO), num contexto de expansão das políticas sociais (BRASIL, 2004; PUCCA JR et al., 2015).

A PNSB expressa, por meio de suas diretrizes, a intenção de induzir um renovado processo de trabalho pelas Equipes de Saúde Bucal (ESB), utilizandose da epistemologia da Saúde Coletiva, em defesa da produção do cuidado e da promoção de saúde, como contraponto a uma prática odontológica a ser superada, caracterizada como ultraespecializada, centrada em procedimentos tecnificados e mecanicista (BOTAZZO, 2013; BRASIL, 2004).

As referências à "promoção da saúde" aparecem de forma transversal na PNSB, como requisitos para adequação no processo de trabalho, no âmbito da intersetorialidade (envolvimento das escolas, locais de trabalho, comércio, mídia, indústria, dentre outros, nas ações de saúde), como pressuposto para medidas que almejem redução dos fatores de risco e também educativo-preventivas (BRASIL, 2004). A PNSB declara que a "promoção de saúde bucal" faz parte de um conceito amplo e transcendente das dimensôes técnicas do setor odontológico, se relacionando à construção de políticas públicas saudáveis como acesso à água tratada e fluoretada, aos dentifrícios, cuidados odontológicos básicos, abordagem de fatores de risco comuns, políticas de alimentação, tabagismo, acidentes, dentre outros (BRASIL, 2004). Destacamos ainda, nesse âmbito, a manifestação da defesa da autonomia dos cidadáos, indicando a necessidade de esforços para estimular práticas de autocuidado e combate a medicalização e dependência excessiva dos profissionais ou serviços de saúde (BRASIL, 2004).

Tal política avança também no sentido da integralidade, ao extrapolar a tradição de assistência odontológica pública restrita às crianças, característica do Sistema Incremental, marcada pela renúncia da oferta de procedimentos de maior 
complexidade, como próteses e determinados tipos de cirurgia, realizados a partir de então nos CEO (BRASIL, 2004; CHAVES, 1977).

A expansão do número de ESB em todo o território nacional, assim como o incremento na complexidade dos propósitos da odontologia nesse novo paradigma, agrega desafios para a gestão. Espera-se que a qualificação e a expansão da oferta de serviços sejam acompanhadas não só por uma melhoria no quadro de morbidade da população assistida, mas também por ganhos de cidadania, educação em saúde e incremento nos níveis de autonomia dos usuários (FLEURY-TEIXEIRA, 2008). Existe, portanto, a necessidade de produçáo de informaçóes alinhadas a esse novo cenário, que sirvam como ferramentas para os níveis de macro, meso e microgestão em saúde (SOUZA, 2009).

Os indicadores de saúde são ferramentas importantes para a gestão pública, conceituados como medidas que sintetizam informaçóes sobre atributos e dimensôes por vezes abstratas, do processo saúde-doença e dos serviços, que servem ao propósito de possibilitar mensuraçôes e avaliaçôes (OPAS, 2018). Podem ser comparados a fotografias, que retratam em diferentes perspectivas, aspectos distintos da realidade, ou seja, a traduz num modelo, indicando informações relevantes para a condução de políticas públicas (JANUZZI, 2012).

Os indicadores tradicionalmente utilizados para a abordagem de questôes odontológicas tratam da mensuração de condiçôes bucais, de acesso e da utilização dos serviços, como índices de cárie, doenças periodontais, má-oclusão, cobertura de primeira consulta odontológica programática, tratamento concluído, dentre outros (CHAVES, 1977; LUIZ et al. 2008).

Esses indicadores foram concebidos dentro de um paradigma odontológico tradicional, que se almeja superar, segundo as diretrizes da PNSB de 2004. Assim, para além dos objetivos de produção de atendimentos e procedimentos, o desenvolvimento de conhecimentos, responsabilização e autonomia agregam-se como fins das intervençôes da equipe de saúde bucal, para os quais acredita-se haver uma carência de indicadores adequados (BRASIL, 2004; BRASIL, 2006).

Esta revisão integrativa tem como objetivo apresentar uma síntese das produçóes científicas a respeito do uso de indicadores pela gestão em odontologia no Brasil, no contexto do SUS, nos últimos 15 anos, para assim, colaborar com o apontamento e sugestão de lacunas a serem exploradas em futuras pesquisas. Partimos da seguinte questão de pesquisa: os indicadores de serviços utilizados por pesquisas que tiveram 
como objeto os serviços odontológicos no SUS, no contexto da PNSB, são coerentes com os objetivos declarados dessa política ou se restringem ao escopo tradicional de programação dos serviços?

\section{Fontes e métodos}

Este trabalho foi feito através de uma revisão integrativa da literatura, método que objetiva sistematizar e sintetizar resultados de pesquisas dentro de um tema delimitado, interconectando elementos isolados de outros estudos (ROMAN, 1998; MENDES et al, 2008). Segundo Mendes et al (2008), tal método permite avaliar as buscas de forma crítica e sintetizar evidências sobre o tema investigado, produzindo um panorama do estado atual do conhecimento, sugerindo a implementação de intervenções e identificando lacunas que subsidiarão futuros estudos.

O percurso metodológico desta pesquisa compreendeu seis passos, conforme preconizado por Mendes et al (2008): 1- identificação do tema e elaboração de questấo norteadora; 2- definição e declaração de critérios para inclusão e exclusão de estudos/amostragem ou busca na literatura; 3- definição das informaçôes a serem extraídas dos estudos; 4- avaliação dos estudos incluídos; 5- interpretação dos resultados e 6- apresentação da revisão/síntese do conhecimento.

Os descritores utilizados nas buscas foram eleitos por meio da consulta na Biblioteca Virtual em Saúde, tendo sido utilizada a combinação dos seguintes termos: Saúde Bucal, Odontologia, Sistema Único de Saúde, Indicadores, com os complementos "de Serviço", "de Gestão", "Básicos de Saúde," e "de Qualidade em Assistência à Saúde" (BVS, 2018).

Foram definidos, como critérios de inclusão, artigos provenientes de dados primários e/ou secundários, publicados entre os anos de 2004 a 2018, disponíveis online e referentes ao âmbito brasileiro. Foram excluídos os artigos provenientes da busca cuja temática não se relacionava com a pergunta de pesquisa.

As buscas foram feitas nas bases Scielo e Bireme, resultando inicialmente em 123 artigos. Com o apoio do software online "My Endnote Web", foram identificados os repetidos, resultando em 33 artigos para leitura de títulos, que permitiu a exclusão daqueles cuja temática era claramente distinta do escopo desta revisão, tendo permanecido 29 artigos para leitura de resumos. Desses, 21 artigos permaneceram para leitura na íntegra, compondo a amostra final. 
Inicialmente, foi realizada leitura flutuante, a fim do pesquisador impregnar-se dos sentidos dos textos e obter uma compreensão panorâmica, seguida por leitura estruturada com vistas à elaboração de fichamento, atenta aos seguintes itens: título do artigo, autores, tipo do estudo, objetivos, indicadores utilizados como ferramenta ou como objeto de discussão, resultados principais e conclusões.

O produto dessa análise inicial dos artigos consiste na organização e descrição dos itens supracitados (Quadro 1) e complementa-se pela construção de categorias a posteriori, em seguida apresentadas e discutidas.

\section{Resultados}

Os critérios de busca, bem como os descritores selecionados, mostraram-se adequados ao elegerem artigos cujos conteúdos permitiram abordar os objetivos propostos para esta revisão integrativa de literatura.

\section{Quadro 1. Artigos incluídos na revisão}

\begin{tabular}{|l|l|l|}
\hline Autores & Ano & Título \\
\hline França et al & 2018 & $\begin{array}{l}\text { Oral health indicators in the Interfederative Pacts of the Unified } \\
\text { Health System: development in the 1998-2016 period }\end{array}$ \\
\hline Gonçalves et al & 2018 & $\begin{array}{l}\text { Desempenho dos Centros de Especialidades Odontológicas da 2a } \\
\text { Regional de Saúde do Paraná }\end{array}$ \\
\hline Silva et al & 2018 & $\begin{array}{l}\text { Caracterização do modelo de atenção básica à saúde bucal na região } \\
\text { nordeste no período de 2015-2017 }\end{array}$ \\
\hline Almeida et al & 2016 & $\begin{array}{l}\text { Acesso ao implante dentário osteointegrado no Sistema Único de } \\
\text { Saúde (SUS): descriçáo do panorama nacional. }\end{array}$ \\
\hline Fernandes et al & 2016 & $\begin{array}{l}\text { Avaliação dos indicadores de saúde bucal no Brasil: tendência } \\
\text { evolutiva pró-equidade? }\end{array}$ \\
\hline Aguiar \& Celeste & 2015 & $\begin{array}{l}\text { Necessidade e alocação de laboratórios regionais de prótese dentária } \\
\text { no Brasil: um estudo exploratório }\end{array}$ \\
\hline Pucca Jr et al & 2015 & $\begin{array}{l}\text { Ten Years of a National Oral Health Policy in Brazil: Innovation, } \\
\text { Boldness, and Numerous Challenges }\end{array}$ \\
\hline Bonfante et al & 2014 & $\begin{array}{l}\text { Sobrevida de cinco anos e fatores associados ao câncer de boca para } \\
\text { pacientes em tratamento oncológico ambulatorial pelo Sistema } \\
\text { Único de Saúde, Brasil }\end{array}$ \\
\hline
\end{tabular}

continua... 


\begin{tabular}{|c|c|c|}
\hline Autores & Ano & Título \\
\hline Cortelazzi et al & 2014 & $\begin{array}{l}\text { Variáveis associadas ao desempenho de Centros de Especialidades } \\
\text { Odontológicas no Brasil }\end{array}$ \\
\hline $\begin{array}{l}\text { Figueiredo } \& \\
\text { Briáo }\end{array}$ & 2014 & $\begin{array}{l}\text { Atendimento Odontológico às Gestantes do Município de Rio } \\
\text { Grande, Rio Grande do Sul }\end{array}$ \\
\hline Pimentel et al & 2014 & $\begin{array}{l}\text { A utilização dos indicadores de saúde bucal e de desenvolvimento } \\
\text { humano no monitoramento da atenção básica nos municípios da } \\
\text { região metropolitana de Curitiba-PR }\end{array}$ \\
\hline Zermiani et al & 2014 & $\begin{array}{l}\text { Indicadores de desenvolvimento humano e de saúde bucal na atenção } \\
\text { básica nos municípios da regiấo metropolitana de Curitiba-PR }\end{array}$ \\
\hline Neves et al & 2013 & $\begin{array}{l}\text { Variáveis sociodemográficas e psicocomportamentais associadas à } \\
\text { gengivite e à pobre higiene bucal em pacientes de uma unidade de } \\
\text { saúde da família }\end{array}$ \\
\hline Silva et al & 2013 & $\begin{array}{l}\text { Indicadores de atenção básica e especializada em saúde bucal nos } \\
\text { municípios do Estado do Pará, Brasil: estudo ecológico, 2001-2010 }\end{array}$ \\
\hline Bordin \& Fadel & 2012 & $\begin{array}{l}\text { Pacto pela saúde no Brasil: uma análise descritiva da progressão dos } \\
\text { indicadores de saúde bucal }\end{array}$ \\
\hline Guimarães et al & 2011 & $\begin{array}{l}\text { Associação entre as açôes de serviços de saúde bucal, Índice de } \\
\text { desenvolvimento Humano, presença de água fluoretada e cárie } \\
\text { dentária em crianças aos cinco anos de idade }\end{array}$ \\
\hline Lira Júnior et al & 2011 & Indicadores socioeconômicos e desigualdades em Saúde Bucal no Brasil \\
\hline Lessa \& Vettore & 2010 & $\begin{array}{l}\text { Gestão da Atenção Básica em Saúde Bucal no Município de } \\
\text { Fortaleza, Ceará, entre } 1999 \text { e } 2006\end{array}$ \\
\hline Nickel et al & 2009 & Modelo de Avaliação da Atenção em Saúde Bucal \\
\hline Lucietto et al & 2008 & $\begin{array}{l}\text { Revisão e discussão sobre indicadores para a previsão de demanda } \\
\text { por cirurgiốes-dentistas no Brasil }\end{array}$ \\
\hline Fernandes \& Peres & 2005 & $\begin{array}{l}\text { Associaçáo entre atenção básica em saúde bucal e indicadores } \\
\text { socioeconômicos municipais }\end{array}$ \\
\hline
\end{tabular}

É possível observar a diversidade de indicadores usados desde os anos iniciais da PNSB, abordando uma ampla gama de temas, como mensurações epidemiológicas, distribuição de recursos e infraestrutura relativos a serviços de saúde bucal, dentre outros (Quadro 2) (Brasil, 2004). Optou-se por categorizá-los segundo a divisão proposta por Donabedian (1988): entre indicadores de estrutura, processo e resultado. Neste último, foram considerados também os indicadores de estado de 
saúde, embora sejam produto não apenas do desempenho dos serviços de saúde, mas também de uma complexa rede de causalidade.

A maioria dos artigos valeu-se de dados secundários, sobretudo, oriundos do Departamento de Informática do SUS (DATASUS), além de dados socioeconômicos provenientes do Instituto Brasileiro de Geografia e Estatística (IBGE) e Programa das Naçôes Unidas para o Desenvolvimento (PNUD) (DATASUS, 2019; PNUD, 2019; IBGE, 2004).

Quadro 2. Síntese de indicadores utilizados nos artigos incluídos na revisão e sua classificação segundo Donabedian (1988).

\begin{tabular}{|c|c|c|c|}
\hline Indicador & Estrutura & Processo & Resultado \\
\hline $\begin{array}{l}\text { LRPD* credenciados por macrorregião brasileira ou por } \\
\text { cidade. }\end{array}$ & $\mathrm{X}$ & & \\
\hline TPDs** vinculados ao SUS por macrorregião brasileira. & $\mathrm{X}$ & & \\
\hline Dentistas com vínculo SUS, profissional por população. & $\mathrm{X}$ & & \\
\hline Indicador epidemiológico de edentulismo. & & & $\mathrm{X}$ \\
\hline Indicador epidemiológico de uso e necessidade de próteses. & & & $\mathrm{X}$ \\
\hline Taxa de produção mensal de próteses. & & $\mathrm{X}$ & \\
\hline Implantes osteointegrados por mil habitantes. & & $\mathrm{X}$ & \\
\hline Indicador epidemiológico de necessidade de implantes. & & & $\mathrm{X}$ \\
\hline $\begin{array}{l}\text { Sobrevida global e específica de pacientes diagnosticados } \\
\text { com câncer bucal. }\end{array}$ & & & $\mathrm{X}$ \\
\hline $\begin{array}{l}\text { Número de equipes que desempenharam açóes } \\
\text { relacionadas a câncer bucal. }\end{array}$ & & $\mathrm{X}$ & \\
\hline Cobertura populacional de ESF e Atenção Básica. & $\mathrm{X}$ & & \\
\hline Cobertura de escovação dentária supervisionada & & $\mathrm{X}$ & \\
\hline Produção de procedimentos especializados & & $\mathrm{X}$ & \\
\hline Tempo de implantação, tipo e número de CEOs instalados. & $\mathrm{X}$ & & \\
\hline Cobertura e produção de primeira consulta. & & $\mathrm{X}$ & \\
\hline Produção de procedimentos odontológicos básicos. & & $\mathrm{X}$ & \\
\hline $\begin{array}{l}\text { Proporçáo de atendimentos odontológicos especializados } \\
\text { sobre básicos. }\end{array}$ & & $\mathrm{X}$ & \\
\hline
\end{tabular}

continua... 


\begin{tabular}{|c|c|c|c|}
\hline Indicador & Estrutura & Processo & Resultado \\
\hline Cobertura populacional de Equipes de Saúde Bucal. & $\mathrm{X}$ & & \\
\hline Procedimentos coletivos. & & $\mathrm{X}$ & \\
\hline $\begin{array}{l}\text { Proporção exodontias sobre demais procedimentos } \\
\text { individuais na } \mathrm{AB} \text {. }\end{array}$ & & $\mathrm{X}$ & \\
\hline Proporção de gestantes em atendimento odontológico. & & $\mathrm{X}$ & \\
\hline Número de consultas odontológicas de gestantes. & & $\mathrm{X}$ & \\
\hline $\begin{array}{l}\text { Número de equipes de SB que desempenharam açóes para } \\
\text { gestantes. }\end{array}$ & $\mathrm{X}$ & & \\
\hline Indicador epidemiológico de cárie CPO-D e ceo-d. & & & $\mathrm{X}$ \\
\hline Indicador epidemiológico índice de placa e índice gengival. & & & $\mathrm{X}$ \\
\hline Proporção de população coberta por água fluoretada. & $\mathrm{X}$ & & \\
\hline $\begin{array}{l}\text { Presença ou não da SB no relatório da conferência } \\
\text { municipal de saúde. }\end{array}$ & & $\mathrm{X}$ & \\
\hline $\begin{array}{l}\text { Proporção de unidades de saúde que tiveram interrupçáo } \\
\text { de mais de dois dias consecutivos por falta de profissional. }\end{array}$ & $\mathrm{X}$ & & \\
\hline $\begin{array}{l}\text { Proporção de unidades de saúde que tiveram interrupçáo } \\
\text { de mais de dois dias consecutivos para manutenção ou } \\
\text { conserto de equipamentos. }\end{array}$ & $\mathrm{X}$ & & \\
\hline $\begin{array}{l}\text { Proporção de unidades de saúde que realizaram grupos de } \\
\text { educaçâo com atividades de Saúde Bucal. }\end{array}$ & & $\mathrm{X}$ & \\
\hline $\begin{array}{l}\text { Presença ou não de campo para anotação de exame de } \\
\text { mucosa na ficha clínica. }\end{array}$ & $\mathrm{X}$ & & \\
\hline $\begin{array}{l}\text { Presença ou náo de unidade com atendimento no terceiro } \\
\text { turno. }\end{array}$ & $\mathrm{X}$ & & \\
\hline Porcentagem de unidades que realizaram visita domiciliar & & $\mathrm{X}$ & \\
\hline $\begin{array}{l}\text { Porcentagem de procedimentos específicos de prótese } \\
\text { dentária }\end{array}$ & & $\mathrm{X}$ & \\
\hline Investimento em Saúde Bucal no SUS & $\mathrm{X}$ & & \\
\hline Número de Equipes de Saúde Bucal na Atenção Básica & $\mathrm{X}$ & & \\
\hline
\end{tabular}

*LRPD - Laboratório Regional de Prótese Dentária

**TPD - Técnico de Prótese Dentária 
A frequência do uso de tais indicadores foi mensurada nos estudos revisados, e em seguida foram elaboradas categorias, a fim de agrupar os indicadores por meio de seus atributos centrais (Tabela 1).

Tabela 1. Frequência e proporção de indicadores presentes nos estudos.

\begin{tabular}{l|c|c}
\hline Tipo de indicador & $\mathbf{n}$ & $\%$ \\
\hline Produtividade & 36 & 41 \\
\hline Capacidade instalada & 24 & 27 \\
\hline Primeira consulta odontológica & 9 & 10 \\
\hline Indicadores epidemiológicos & 9 & 10 \\
\hline Indicadores de açốes estratégicas & 8 & 9 \\
\hline Indicadores sobre interdiçóes de funcionamento do serviço de SB & 2 & 2 \\
\hline Indicador de participaçáo social & 1 & 1 \\
\hline TOTAL & 89 & 100 \\
\hline
\end{tabular}

Por fim, foi analisado se os temas-chave, constituídos por diretrizes, pressupostos e objetivos manifestados nos documentos da PNSB e no Caderno 17 da Atenção Básica, principais marcos norteadores do modelo brasileiro de política pública de Saúde Bucal, são contemplados pelos indicadores identificados na busca (Quadro 3).

Quadro 3. Temas-chave oriundos da PNSB e Caderno 17 da AB e correspondência nos indicadores identificados nos estudos.

\begin{tabular}{|l|c|c|}
\hline \multirow{2}{*}{$\begin{array}{l}\text { Temas-chave presentes na PNSB e Caderno 17 da } \\
\text { Atençáo Básica }\end{array}$} & Sim & Náo \\
\cline { 2 - 3 } & & $\mathrm{X}$ \\
\hline Qualidade na Atenção Básica & & $\mathrm{X}$ \\
\hline Resolutividade na Atenção Básica & $\mathrm{X}$ & $\mathrm{X}$ \\
\hline Funcionamento em rede & $\mathrm{X}$ & \\
\hline $\begin{array}{l}\text { Integralidade (promoção, proteção, prevenção, } \\
\text { tratamento, cura e reabilitação, individual e coletivo) }\end{array}$ & $\mathrm{X}$ & \\
\hline Uso de epidemiologia & \multicolumn{2}{|}{} \\
\hline Vigilância em saúde & & \\
\hline
\end{tabular}

continua... 


\begin{tabular}{|c|c|c|}
\hline \multirow{2}{*}{$\begin{array}{l}\text { Temas-chave presentes na PNSB e Caderno } 17 \text { da } \\
\text { Atençáo Básica }\end{array}$} & \multicolumn{2}{|c|}{ Contemplado por indicadores nos estudos } \\
\hline & Sim & Não \\
\hline Ênfase na ESF & $\mathrm{X}$ & \\
\hline Educação permanente, formação & & $\mathrm{X}$ \\
\hline Financiamento & $\mathrm{X}$ & \\
\hline Definir agenda de pesquisa & & $\mathrm{X}$ \\
\hline Gestão participativa & $\mathrm{X}$ & \\
\hline Ética & & $\mathrm{X}$ \\
\hline Acesso & $\mathrm{X}$ & \\
\hline Acolhimento usuário-centrado, humanização & & $\mathrm{X}$ \\
\hline Vínculo & & $\mathrm{X}$ \\
\hline Responsabilidade profissional & & $\mathrm{X}$ \\
\hline Interdisciplinaridade e multiprofissionalismo & & $\mathrm{X}$ \\
\hline Intersetorialidade & & $\mathrm{X}$ \\
\hline $\begin{array}{l}\text { Proporção de horas do CD para assistência e demais } \\
\text { atividades }\end{array}$ & & $\mathrm{X}$ \\
\hline Garantir e qualificar atendimento de urgência & & $\mathrm{X}$ \\
\hline Condições de trabalho & & $\mathrm{X}$ \\
\hline Açóes de promoção e proteção de Saúde & $\mathrm{X}$ & \\
\hline Fluoretação das águas & $\mathrm{X}$ & \\
\hline Educação em Saúde & & $\mathrm{X}$ \\
\hline Higiene Bucal Supervisionada & $\mathrm{X}$ & \\
\hline Aplicação tópica de flúor & & $\mathrm{X}$ \\
\hline Diagnóstico e tratamento de doenças e lesões & $\mathrm{X}$ & \\
\hline Açôes de reabilitação & $\mathrm{X}$ & \\
\hline Medidas pra prevenção e controle do câncer bucal & $\mathrm{X}$ & \\
\hline Inclusão de procedimentos mais complexos na $\mathrm{AB}$ & $\mathrm{X}$ & \\
\hline Inclusão da reabilitação protética na $\mathrm{AB}$ & & $\mathrm{X}$ \\
\hline Garantia de acesso e educação em saúde para gestantes & $\mathrm{X}$ & \\
\hline
\end{tabular}

continua... 


\begin{tabular}{|l|c|c|}
\hline \multirow{2}{*}{$\begin{array}{l}\text { Temas-chave presentes na PNSB e Caderno 17 da } \\
\text { Atençáo Básica }\end{array}$} & \multicolumn{2}{|c|}{ Contemplado por indicadores nos estudos } \\
\cline { 2 - 3 } & Sim & Não \\
\hline Dispor de horários compatíveis para adultos & & $\mathrm{X}$ \\
\hline Favorecer fluxo de acesso para idosos & $\mathrm{X}$ & \\
\hline Ampliação e qualificação atenção secundária e terciária & & $\mathrm{X}$ \\
\hline Fortalecimento da autonomia dos indivíduos & & $\mathrm{X}$ \\
\hline Fortalecimento de ações comunitárias & & $\mathrm{X}$ \\
\hline Abordagem por fatores de risco comuns & & \\
\hline
\end{tabular}

\section{Discussão}

Os indicadores são ferramentas que tornam possíveis o monitoramento e avaliação de aspectos de interesse para a gestão de políticas públicas, a partir da mensuração de atributos que traduzem dados de relevância social (FRANÇA et al, 2018). Segundo Malmegrin (2014), os indicadores permitem organizar informaçóes desordenadas de diversas fontes, produzindo um item de síntese informacional. Ao fornecer uma representação simplificada de determinada face da realidade, subsidiam o gestor, informando o Ciclo de Shewhart ("plan, do, check, act"- PDCA) (JANUZZI, 2012).

Januzzi (2012) faz uma analogia entre os indicadores e as fotografias, no sentido de que tratam-se de informaçóes a princípio estáticas, que revelam sempre uma determinada parte da realidade, escolhida segundo interesses e perguntas socialmente definidas. Pressupóe-se que um conjunto de fotografias ou indicadores, feitos a partir de diferentes perspectivas, amplie a capacidade de representação da realidade.

A diversidade de aspectos representados nos indicadores encontrados demonstra a pluralidade de perspectivas e indagaçóes acerca do tema pesquisado. A organização dos resultados em termos de categorias, frequência e proporção de utilização permite tecer uma análise acerca dos aspectos mais valorizados pelos estudos, bem como apontar lacunas tendo como referência as principais diretrizes da PNSB e do Caderno 17 da Atenção Básica (BRASIL, 2004; 2006).

Assim, o conjunto de indicadores pôde ser tipificado em sete categorias: indicadores de produtividade, de capacidade instalada, de acesso a primeira consulta odontológica programática, epidemiológicos, de ações estratégicas, sobre interdições de funcionamento do serviço de SB e de participação social. 


\section{Indicadores de produtividade}

Os indicadores de produtividade foram aqueles mais frequentes nos estudos (41\% do total), sendo eles: taxa de produção mensal de próteses, de implantes osteointegrados por regiáo, cobertura de escovação dentária supervisionada, produção de procedimentos especializados, produçáo de procedimentos odontológicos básicos, produção de procedimentos coletivos, proporção de exodontias sobre demais procedimentos individuais na $\mathrm{AB}$ e procedimentos preventivos.

O fato de terem sido utilizados indicadores relacionados às necessidades e produção de próteses dentárias, implantes e procedimentos especializados é relevante ao se considerar que, antes da PNSB (BRASIL, 2004), foi preponderante na história do modelo estatal de serviços odontológicos a assistência restrita aos escolares e limitada a procedimentos clínicos básicos e mutiladores (NARVAI, 2006). Aparentemente, existe uma preocupação com o monitoramento e avaliação de necessidades e de produção de procedimentos de elevado nível de complexidade e de uma faixa etária incorporados pela PNSB (BRASIL, 2004).

O indicador de escovação dentária supervisionada, segundo Nota Técnica do Ministério da Saúde (BRASIL, 2010), é considerado um indicador de cobertura de usuários que tiveram acesso a esse tipo de medida preventiva. Apesar dessa nota esclarecer sobre a dimensão educativa do conceito que subjaz essa ação e orientar sobre a necessidade de as equipes de saúde bucal evitarem atitudes de fiscalização, sançóes, ameaças e julgamentos do tipo "certo-errado ou bom-mau", ponderamos que se trata de um indicador impreciso, por contemplar, indistintamente, tanto atividades produtoras de autonomia quanto aquelas social e culturalmente iatrogênicas. Segundo Illich (1975), tais iatrogenias ocorrem na medida em que determinadas tecnologias profissionais reduzem a autonomia das pessoas e da sociedade sobre questôes triviais, como, no caso, o autocuidado higiênico, tornando-se dependentes de saberes especializados. Nesse sentido, atribui-se ao campo odontológico o fenômeno do fetichismo pela escovação e higiene bucal, ou seja, a necessidade de promover técnicas odontológicas e especializadas de higiene e produtos do tipo mercadorias (escovas, pastas, fios) passa a ser mais importante do que o próprio ganho de coeficientes de autonomia e saúde (LEME et al, 2019; BOTAZZO 2013). Dessa forma, não negamos a importância ou utilidade desse indicador, mas defendemos a necessidade de ponderar seu significado, sobretudo porque é referenciado de forma pouco refletida. 
Os indicadores sobre os tipos e relaçôes entre procedimentos realizados (preventivos, curativos e mutiladores) refletem a preocupação de mensurar as taxas de produção e de avaliar se o modelo de atenção em saúde bucal tem conseguido inverter uma lógica mutiladora e oferecer açóes no sentido da integralidade, que seria constatável pelo predomínio de ações preventivas (aplicação tópica de flúor, aplicaçôes de selantes de fóssulas e fissuras, etc) e curativas (dentística restauradora, tratamento endodôntico, etc), sobre aquelas mutiladoras (exodontias). Apesar de reconhecer o valor desses indicadores, novamente defendemos a necessidade de ponderação acerca de seus significados, uma vez que sob a denominação de procedimentos preventivos e coletivos, podem estar contidas práticas produtoras de dependência e indutoras de um consumismo irracional de produtos de higiene bucal, assim como é sabido que a ênfase em práticas curativas, sobretudo pautadas na dentística restauradora, é uma das razóes para desfechos mutiladores, segundo Elderton (2003).

\section{Indicadores de capacidade instalada e cobertura de primeira consulta odontológica programática}

O grupo de indicadores de capacidade instalada foi o segundo mais frequente na literatura analisada (27\%), composto pela agregação dos indicadores: LRPD credenciados por macrorregião brasileira ou por cidade, TPDs vinculados ao SUS por macrorregião brasileira, número de dentistas nos municípios e no SUS, cobertura populacional de ESF ou $\mathrm{AB}$, tempo de implantação do $\mathrm{CEO}$, tipo de $\mathrm{CEO}$, número de $\mathrm{CEO}$ instalados no país, proporção de atendimentos odontológicos especializados sobre básicos, cobertura populacional de ESB, população coberta por água fluoretada, existência de unidade com atendimento no terceiro turno, investimento em SB no SUS, número de ESB na AB.

Tais indicadores representam aspectos importantes dos níveis estratégico e tático de planejamento da PNSB, por se relacionarem com produtos da formulação e financiamento dessa política pública em nível federal, que acabam por induzir a organização do sistema no nível municipal (ROSA, 2014). É esse grupo de indicadores que mais evidentemente expóe, provavelmente, os grandes avanços estruturais logrados com a PNSB, reunindo resultados de longo e médio prazo para informar o Ciclo de Shewhart (PDCA) com aplicabilidade, principalmente, para os níveis da média e alta gestão do sistema (PUCCA JR, 2015). 
O indicador de primeira consulta odontológica programática, apesar de se relacionar com os indicadores de capacidade instalada, foi considerado a parte em decorrência de sua importância singular: é produto da gestão estratégica e tática mas também da operacional, uma vez que depende do grau e das formas de organização da atenção em saúde bucal no nível das equipes locais, que muitas vezes se encarregam de organizar o fluxo de acesso ao odontológico nas unidades de saúde, como o controle de agenda por exemplo (REIS et al., 2015). Outro argumento acerca de sua pertinência e valor diz respeito à dívida histórica decorrente dos modelos estatais de saúde bucal, excludentes e elitistas até poucas décadas atrás, sendo a ampliação do acesso ao cuidado programático um objetivo central a ser perseguido a partir da PNSB de 2004 (PUCCA JR, 2015; NARVAI, 2006; BOTAZZO, 2013).

\section{Indicadores epidemiológicos}

Esse grupo de indicadores, composto pela prevalência de edentulismo, uso e necessidade de prótese, de implantes osteointegrados, sobrevida global e específica de pacientes diagnosticados com câncer bucal, índice de cárie CPO-D, índice de placa e gengival, possui a característica de representar, diretamente, mensuraçôes dos níveis de saúde-doença pelo paradigma da epidemiologia. Entendemos que seu uso pelo campo da gestão deve ser cauteloso, considerando a determinação multifatorial (condiçôes socioeconômicas, culturais, ambientais e biológicas) na produção de saúde-doença (BUSS \& PELLEGRINI FILHO, 2007). De qualquer forma, trata-se de uma dimensão importante a ser considerada, pois a redução da carga de morbidades bucais é um dos efeitos desejados de uma política pública efetiva.

\section{Indicadores de açóes estratégicas}

Nessa categoria estão presentes os indicadores número de equipes que desempenharam açóes de câncer bucal, presença de campo para anotação de exame de mucosa na ficha clínica, proporção de gestantes em atendimento odontológico, número de consultas odontológicas de gestantes, número de equipes de SB que desempenharam açóes para gestantes, percentual de unidades de saúde que realizaram grupos de educação com atividades de SB e porcentagem de unidades que realizaram visitas domiciliares. 
A temática do câncer bucal aparece em destaque tanto nas diretrizes da PNSB, quanto no Caderno 17 da $A B$, sendo advogada sua importância por meio da apresentação de dados de morbimortalidade e de altas taxas de diagnósticos em estágios já avançados da doença, a despeito da visibilidade de lesões durante exames físicos que podem ser realizados pelos cirurgiōes dentistas (BRASIL 2004; 2006). Consideramos que o uso de indicadores neste tema é fundamental fonte de subsídio para relevantes políticas neste impactante problema de saúde pública (INCA, 2019).

Outro foco estratégico nas diretrizes da PNSB é a atuação das equipes de saúde bucal junto ao grupo de gestantes, tema contemplado por indicadores próprios nos estudos incluídos nessa revisão (BRASIL, 2004). O Caderno 17 da AB (BRASIL, 2006) coloca que:

A gestação é um acontecimento fisiológico, com alteraçóes orgânicas naturais, mas que
impóe aos profissionais da saúde a necessidade de conhecimentos para uma abordagem
diferenciada. (...) Todo serviço de saúde deve estabelecer, como rotina, a busca ativa das
gestantes de sua área de abrangência (...)

Quanto ao tema da educação em saúde bucal, dentre eles a realização de grupos, confunde-se com a própria história das açôes estatais no campo odontológico (NARVAI, 2006). Inicialmente concebidas e dedicadas aos escolares, o imperativo da necessidade de educar a população acerca de temas de higiene bucal, o "jeito certo de limpar os dentes" (BRASIL, 2006), parece estar impregnado no modus operandi odontológico e defendemos a necessidade de problematização, no sentido de que tais práticas não necessariamente contribuem para o ganho de autonomia e/ ou melhorias nos níveis de autocuidado, considerando a complexidade de fatores que concorrem para a determinação destes objetivos (LEME et al, 2019; BOTAZZO, 2013). De qualquer forma, a presença deste indicador é coerente com as perspectivas contidas nos manuais normativos (BRASIL 2004; 2006).

A presença do indicador relativo às visitas domiciliares pelas equipes de saúde bucal na busca é coerente com a proposição de qualificação e ampliação destas açôes na ESF, a serem desempenhadas, prioritariamente, junto aos acamados ou àqueles com locomoção limitada, em prol da identificação de riscos à saúde bucal e da oferta de acompanhamento e tratamento, neste contexto (BRASIL, 2004). Essa modalidade de atendimento é prevista na organização das demandas assistenciais no enquadre da Atenção Básica (BRASIL, 2006). 


\section{Indicadores sobre interdiçóes de funcionamento do serviço de SB}

Foram contemplados indicadores sobre a paralisação dos serviços odontológicos por questóes de recursos humanos e manutenção de equipamentos (percentual de unidades de saúde que tiveram interrupção de mais de dois dias consecutivos por falta de profissional ou para manutenção de equipamentos).

Por uma perspectiva empírica oriunda do cotidiano da gestão, colocamos que são indicadores importantes para subsidiar o planejamento de medidas que previnam a interrupção da prestação de serviços odontológicos. As atividades odontológicas empregam um amplo arsenal de equipamentos de variados graus de complexidade tecnológica, exigentes de manutenção constante por profissionais especializados. Considerando a dimensão da rede de serviços, severamente expandida após 2004 (PUCCA JR, 2015), certamente tratam-se de relevantes informaçôes para os gestores.

\section{Indicador de participação social}

A gestão participativa é um dos princípios norteadores das ações de saúde bucal, segundo as diretrizes da PNSB (Brasil, 2004), além de ser um dos fundamentos da própria Atenção Básica (BRASIL, 2006). No entanto, nas buscas foi identificada apenas a incipiente presença de um indicador relativo à essa questão: presença ou não da SB no relatório da conferência municipal de saúde.

As conferências municipais de saúde seriam um dos mecanismos de controle social, onde diferentes segmentos da sociedade, como usuários, gestores, prestadores de serviço e trabalhadores da saúde poderiam interferir no planejamento e fiscalização das açốes governamentais, sendo debatidos os diferentes interesses sob o tema da saúde (LIMA \& LIMA, 2017).

A escassez de indicadores nessa dimensão pode ser interpretada como uma necessidade de fortalecimento, na agenda de pesquisa, de temas concernentes ao controle social de políticas de saúde bucal, sobretudo considerando a já mencionada dívida histórica do campo frente à sociedade brasileira, reflexo de um passado no qual a omissáo estatal era regra (NARVAI, 2006).

\section{Panorama geral acerca dos temas-chave contemplados ou não pelos indicadores utilizados}

A listagem de temas-chave presentes nos marcos normativos da PNSB (BRASIL, 2004; 2006) permitiu a realização de um julgamento, por parte dos autores, acerca 
da contemplação ou não de tais temas pelos indicadores localizados nos estudos que compuseram a revisão (Quadros 2 e 3). Nota-se que diversos temas-chave, dentre eles a questão da ética, do vínculo, o fortalecimento da autonomia dos indivíduos e das ações comunitárias, são centrais aos objetivos da PNSB, porém não foram contemplados nos estudos analisados (Quadro 3). Nesse sentido, é oportuno retomar a categoria do cuidado segundo as diretrizes da PNSB (BRASIL, 2004, p.3):

\footnotetext{
(...) eixo de reorientação do modelo, respondendo a uma concepção de saúde não centrada somente na assistência aos doentes, mas, sobretudo, na promoçáo da boa qualidade de vida e intervenção nos fatores que a colocam em risco (...) A produção do cuidado traz consigo a proposta de humanização do processo de desenvolver açóes e serviços de saúde (...) construir, com os usuários, a resposta possível às suas dores, angústias, problemas e afliçóes de uma forma tal que náo apenas se produzam consultas e atendimentos, mas que o processo de consultar e atender venha a produzir conhecimento, responsabilização e autonomia em cada usuário.
}

Julgamos necessária a implementação de indicadores que possam, de fato, monitorar e avaliar os efeitos da PNSB nessas dimensôes. O predomínio do uso de indicadores de estrutura e processos, em detrimento dos de resultados, pode ser reflexo do estágio em que a jovem política se encontra, mas levanta a existência dessa lacuna a ser ocupada pela agenda de pesquisa.

Importante a menção de que mesmo considerando-se a totalidade dos indicadores oficiais, declarados nos Pactos da Atenção Básica, pela Saúde, e no Contrato Organizativo da Ação Pública da Saúde, de mensuração e acompanhamento compulsório pelos gestores municipais e/ou estaduais, objetivos da PNSB relacionados a ganhos de autonomia, cidadania e o combate a medicalização, por exemplo, não são contemplados (FERNANDES et al, 2016).

\section{Considerações finais}

Este estudo possui limitaçóes inerentes à restrição de escopo, necessária para a elaboração de qualquer estudo de revisão integrativa, bem como dos descritores e critérios de seleçáo utilizados. Provavelmente, a incorporação de outras bases de dados resultaria na seleção de importantes artigos relacionados à Pesquisa Nacional de Saúde Bucal - SB Brasil 2010 (BRASIL, 2012) É prudente enfatizar que não é possível realizar inferências ou julgamentos acerca do grau de utilização desses indicadores pelos diversos níveis de gestão, por meio do método utilizado nesta revisão, que contemplou artigos oriundos do campo acadêmico. Entende-se que a 
gestão e a academia possuem agendas nem sempre coincidentes e que, certamente, os gestores fazem uso de outros tipos de indicadores não retratados nos artigos.

Contemplando a pergunta de pesquisa que norteou esta revisão, consideramos que os indicadores de serviços utilizados por pesquisas que tiveram como objeto os serviços odontológicos no SUS no contexto da PNSB, embora sejam capazes de representar diferentes faces da realidade e venham sendo utilizados para oportunas análises, desconsideram dimensôes importantes acerca do cuidado e de promoção de saúde, manifestadas no bojo da política, priorizando aspectos tradicionais de programação dos serviços e de avaliação estrutural.

\section{Referências Bibliográficas}

AGUIAR, V.R.; CELESTE, R.K. Necessidade e alocação de laboratórios regionais de prótese dentária no Brasil: um estudo exploratório. Ciência \& Saúde Coletiva, 20(10):3121-3128, 2015. ALMEIDA, A.M.R. et al. Acesso ao implante dentário osteointegrado no Sistema Único de Saúde (SUS): descrição do panorama nacional. Arquivos de Odontologia, Belo Horizonte, 52(3): $145-153$, jul/set 2016.

BONFANTE, G.M.dS. et al. Sobrevida de cinco anos e fatores associados ao câncer de boca para pacientes em tratamento oncológico ambulatorial pelo Sistema Único de Saúde, Brasil. Cadernos de Saúde Pública, Rio de Janeiro, 30(5):983-997, mai, 2014.

BOTAZZO C. Diálogos sobre a boca. São Paulo: Hucitec; 2013.

BORDIN, D.; FADEL, C.B. Pacto pela saúde no Brasil: uma análise descritiva da progressão dos indicadores de saúde bucal. Rev Odontol UNESP. Sept-Oct; 41(5): 305-311, 2012.

BRASIL. Ministério da Saúde. Diretrizes da Política Nacional de Saúde Bucal. Secretaria de Atenção à Saúde. Departamento de Atenção Básica. Coordenação Nacional de Saúde Bucal. Brasília; 2004. - Ministério da Saúde. Saúde bucal. Brasília: Ministério da Saúde. Cadernos de Atenção Básica, no 17. Série A. Normas e Manuais Técnicos. Brasília; 2006.

- Ministério da Saúde. Secretaria de Atenção à Saúde. Nota técnica DAB/CGSB: Indicador Média da Açâo Coletiva de Escovação Dental Supervisionada. Brasília, DF. 2010. Disponível em: <http://189.28.128.100/dab/docs/geral/nota_indicador_escovacao.pdf>. Ministério da Saúde. Secretaria de Vigilância em Saúde. SB Brasil 2010: Pesquisa Nacional de Saúde Bucal: resultados principais. Brasília, DF: SVS; 2012.

. Lei No. 8080/90, de 19 de setembro de 1990. Brasília: DF. 1990. Disponível em <https://www.planalto.gov.br/ccivil_03/LEIS/L8080.htm>. Acesso em: 06 abr. 2019. 
BUSS, P.M.; PELLEGRINI-FILHO, A. Health and its social determinants. Physis 2007; 17(1):77-93.

CHAVES MM. Odontologia Social. 2ed. Rio de Janeiro; Labor; 1977.

CHAVES S.C.L et al. Política Nacional de Saúde Bucal: fatores associados à integralidade do cuidado. Revista de Saúde Pública [Internet]. 2010 Dec [cited 2019 Apr 25] ; 44( 6): 1005-1013. Available from: http://www.scielo.br/scielo.php?script=sci_arttext\&pid=\$00348910201000060 0004\&lng=en. Epub Oct 08, 2010. http://dx.doi.org/10.1590/S0034-89102010005000041.

CORTELLAZZI, K.L. et al. Variáveis associadas ao desempenho de Centros de Especialidades Odontológicas no Brasil. Revista Brasileira de Epidemiologia. Out-Dez 2014; 17(4): 978-988.

Departamento de Informática do SUS: DATASUS. Portal. Disponível em <http://datasus. saude.gov.br/>. Acesso em 01 de mar. 2019.

Descritores em Ciências da Saúde: DeCS. ed. rev. e ampl. São Paulo: BIREME / OPAS / OMS, 2018. Disponível em: < http://decs.bvsalud.org >. Acesso em 01 de mar. 2019.

DONABEDIAN, A. The quality of care: how can it be assessed? Journal of American Medical Association, New York, v. 260, n. 12, p. 1743-1748, Sept. 1988.

ELDERTON R.J. Preventive (evidence-based) approach to quality general dental care. Med Princ Pract. 2003;12(1):12-21. pmid:12707497.

FERNANDES, L.S.; PERES, M.A. Associação entre atenção básica em saúde bucal e indicadores socioeconômicos municipais. Revista de Saúde Pública 2005;39(6):930-6.

FERNANDES, Jd.K.B. et al. Avaliação dos indicadores de saúde bucal no Brasil: tendência evolutiva pró-equidade? Cadernos de Saúde Pública, Rio de Janeiro, 32(2):e00021115, fev, 2016.

FIGUEIREDO, M.C.; BRIĀO, D.V. Atendimento Odontológico às Gestantes do Município de Rio Grande, Rio Grande do Sul. UNOPAR Científica Ciências Biológicas e da Saúde 2014;16(4):335-40.

FLEURY-TEIXEIRA P. et al. Autonomia como categoria central no conceito de promoção de saúde. Ciência \& Saúde Coletiva. 2008;13:2115-22. DOI:10.1590/S1413-81232008000900016

FRANÇA, M.Ad.S. et al. Oral health indicators in the Interfederative Pacts of the Unified Health System: development in the 1998-2016 period. Rev Odontol UNESP. 2018 Jan-Feb; 47(1): 18-24.

GONÇALVES, J.Rd.S.N. et al. Desempenho dos Centros de Especialidades Odontológicas da 2a Regional de Saúde do Paraná. Cadernos Saúde Coletiva, 2018, Rio de Janeiro, 26 (4): 432-438.

GUIMARĀES, M.A.M.B. et al. Associação entre as ações de serviços de saúde bucal, Índice de desenvolvimento Humano, presença de água fluoretada e cárie dentária em crianças aos cinco anos de idade. Arquivos em Odontologia, Belo Horizonte, 47(3): 135-145, jul/set 2011. 
ILLICH, I. A expropriação da saúde. Nêmesis da Medicina. Rio de Janeiro: Nova Fronteira, 1975, 164p.

Instituto Brasileiro de Geografia e Estatística. Pesquisa Nacional de Amostra de Domicílios 2003: microdados. Rio de Janeiro: Instituto Brasileiro de Geografia e Estatística; 2004.

Instituto Nacional do Câncer. Portal. Disponível em <https://www.inca.gov.br/>. Acesso em 01 de mar. 2019.

JANUZZI, P.M. Indicadores socioeconômicos na gestão pública. Florianópolis: Departamento de Ciências da Administração/UFSC; [Brasília]: CAPES:UAB, 2012.

LEME P.A.T. et al. How Brazilian dentists work within a new community care context? A qualitative study. PLoS ONE 14(5): e0216640. https://doi.org/10.1371/journal.pone.0216640. 2019.

LESSA, C.F.M; VETTORE, M.V. Gestão da Atenção Básica em Saúde Bucal no Município de Fortaleza, Ceará, entre 1999 e 2006. Saúde \& Sociedade. São Paulo, v.19, n.3, p.547-556, 2010.

LIMA D.F; LIMA L.A.. O controle social no Sistema Único de Saúde: um olhar crítico à Resolução n. 453/2012. Saúde Debate [serial on the internet]. 2017 11];41(115):1168-76. Available from: http://www.scielo.br/pdf/sdeb/v41n115/0103-1104-sdeb-41-115-1168.pdf

LIRA JÚNIOR, R. et al. Indicadores socioeconômicos e desigualdades em Saúde Bucal no Brasil. Revista Odontológica do Brasil Central 2011;20(52).

LUCIETTO, D.A.; AMÂNCIO FILHO, A.; OLIVEIRA, S.Pd. Revisão e discussão sobre indicadores para a previsão de demanda por cirurgiōes-dentistas no Brasil. Revista da Faculdade de Odontologia de Porto Alegre, Porto Alegre, v. 49, n. 3, p. 28-35, set./dez., 2008.

LUIZ, R.R; COSTA, A.J.L; NADANOVSKY, P. Epidemiologia e Bioestatística em Odontologia. São Paulo: Atheneu, 2008.

MALMEGRIN, M. L. Gestão operacional. 3. ed. rev. atual. - Florianópolis : Departamento de Ciências da Administração / UFSC; [Brasília]: CAPES: UAB, 2014.

MENDES, K.D.S; SILVEIRA R.C.CP; GALVÃO, C.M. Revisão integrativa: método de pesquisa para a incorporação de evidências na saúde e na enfermagem. Texto \& Contexto Enfermagem, vol. 17, núm. 4, octubre-diciembre, 2008, pp. 758-764.

NARVAI, P.C. Saúde bucal coletiva: caminhos da odontologia sanitária à bucalidade. Revista de Saúde Pública. São Paulo, v. 40, n. spe, p.141-7, 2006.

NEVES, P.C.B. et al. Variáveis sociodemográficas e psicocomportamentais associadas à gengivite e à pobre higiene bucal em pacientes de uma unidade de saúde da família. Brazilian Journal of Periodontology - June 2013 - volume 23 - issue 02 - 23(2):16-24.

NICKEL, D.A.; CAETANO, J.C.; CALVO, M.C.M. Modelo de Avaliação da Atenção em Saúde Bucal. Pesquisa Brasileira em Odontopediatria e Clinica Integrada, João Pessoa, 9(3):373-379, set./dez. 2009. 
OPAS - Organização Pan-Americana da Saúde. Indicadores de saúde. Elementos conceituais e práticos. Washington, DC; 2018.

PAIM, J.S. Bases conceituais da reforma sanitária brasileira. In: Fleury S, organizador. A luta do Cebes. São Paulo: Lemos; 1997.

PIMENTEL, B.V. et al. A utilização dos indicadores de saúde bucal e de desenvolvimento humano no monitoramento da atenção básica nos municípios da região metropolitana de Curitiba-PR. Revista Espaço para a Saúde | Londrina | v. 15 | n. 3 | p. $42-52$ | jul./set. 2014.

Programa das Nações Unidas para o Desenvolvimento. Atlas de Desenvolvimento Humano no Brasil. http://www.pnud.org.br/atlas/. Acesso em 01 de mar. 2019.

PUCCA JUNIOR, G.A. et al. Ten years of national oral health policy in Brazil: innovation, boldness, and numerous challenges. Journal of Dental Research. 2015 Oct; 94 (10): 1333-1337.

REIS, W.G.; SCHERER M.D.dA; CARCERERI D.L. O trabalho do Cirurgião-dentista na Atenção Primária à Saúde: entre o prescrito e o real. Saúde debate. 2015; 39(104): 56-64. DOI: 10.1590/0103-110420151040608

ROMAN A.R; FRIEDLANDER, MR. Revisão integrativa de pesquisa aplicada à enfermagem. Cogitare Enfermagem 1998 Jul-Dez; 3(2):109-12.

ROSA, R. A. Gestão Logística. Florianópolis: Departamento de Ciências da Administração/ UFSC; [Brasília]: CAPES: UAB, 2014.

SCHERER C.I.; SCHERER M.D.. Advances and challenges in oral health after a decade of the "Smiling Brazil" Program. Revista de Saude Publica. 2015; 49(98). DOI: 10.1590/S00348910.2015049005961

SILVA, L.S. et al. Indicadores de atenção básica e especializada em saúde bucal nos municípios do Estado do Pará, Brasil: estudo ecológico, 2001-2010. Epidemiologia e Serviços de Saúde, Brasília, 22(2):325-334, abr-jun 2013.

SILVA, S.Ed. et al. Caracterização do modelo de atenção básica à saúde bucal na região nordeste no período de 2015-2017. Archives of Health Investigation (2018) 7(10):402-407.

SOUZA, L.E.P.F.d. O SUS necessário e o SUS possível: gestão. Uma reflexão a partir de uma experiência concreta. Ciência \& Saúde Coletiva, Rio de Janeiro, v. 14, n. 3, p. 911-918, June 2009. Available from <http://www.scielo.br/scielo.php?script=sci_arttext\&pid=S141381232009000300027\&lng=en\&nrm=iso >. access on 06 Apr. 2019. http://dx.doi.org/10.1590/ S1413-81232009000300027

ZERMIANI, T.; PIMENTEL, B.; BUFFON, M.; DITTERICH, R. Indicadores de desenvolvimento humano e de saúde bucal na atenção básica nos municípios da região metropolitana de Curitiba-PR. Revista da Faculdade de Odontologia - UPF, v. 19, n. 2, 2 dez. 2014. 


\section{Abstract}

\section{Oral health indicators in the context of brazilian oral health policy: an integrative review}

Integrative literature review about service indicators related to dentistry in Brazil. We start from the following question: Are the indicators from studies that focused on dental services in SUS either consistent with the health promotion paradigm advocated by the National Oral Health Policy or are they restricted to the traditional scope of service programming? The search was performed in Scielo and Bireme, including articles published between 2004 and 2018, resulting in a final sample of 21 articles, read in full and extracted information about the indicators used, later categorized and analyzed. The results show that, although the diversity of used indicators represent relevant aspects of reality, generally disregard the category of care and attributes of health promotion paradigm, and there is a lack of outcome indicators, which would allow assessing the impact of health policy on user demands.

> Keywords: Oral Health. Dentistry. Unified Health System. Indicators of Health Services. Review. 